\section{Perfil dos idosos freqüentadores de grupos de convivência em Belo Horizonte, Minas Gerais, Brasil}

\author{
A profile of elderly members of community groups \\ in Belo Horizonte, Minas Gerais State, Brazil
}

\section{Abstract}

This study focused on elderly members of community groups, specifically their physical, social, psychological, and economic conditions. A descriptive cross-sectional study included 197 individuals, using the modified Brazilian Old Age Schedule, with questions on physical health, medical services, activities of daily living, social and economic resources, and mental health. The majority of participants were women, widows, and had primary schooling; mean age was 71.66 \pm 6.8 years; $51.27 \%$ considered their health good; $85.28 \%$ reported at least one illness; $63.96 \%$ reported no falls in the previous year; $56.85 \%$ reported urinary incontinence (UI). The majority had attended community groups for the elderly for more than five years; $65.99 \%$ had a monthly income of up to one minimum wage; and $24.37 \%$ displayed depressive symptoms. There was an association between self-perceived health and falls and UI; between falls and neurological, musculoskeletal, and digestive problems and depressive symptoms; between UI and depressive symptoms and activities of daily living; and between family satisfaction and marital status. The health alterations reinforce the epidemiological profile of the elderly and can contribute to orienting collective health measures for this age group.

Aging; Centers of Connivance and Leisure; Health of the Elderly
Paula Lutiene de Castro e Borges 1

Rose Procópio Bretas 1

Silvana Fernandes de Azevedo 1

Juliana Magalhães Machado Barbosa 1

\section{Introdução}

O crescimento da população idosa é um fenômeno mundial 1. No Brasil, em 2000, o Instituto Brasileiro de Geografia e Estatística (IBGE) indicou que 1 em cada 10 brasileiros tinha mais de 60 anos, proporção que deverá atingir 1:5 até 2050. De 1991 a 2000, o número de idosos aumentou em quase 4 milhões, atingindo $8,6 \%$ da população total 2. Em Belo Horizonte, no mesmo período, mais de 204 mil pessoas eram idosas, o equivalente a $9,14 \%$ da população total ${ }^{3}$. Esse aumento acelerado da população idosa ocasiona impacto para diversos setores da sociedade e precisa ser discutido visando a um enfrentamento adequado de suas conseqüências 1,2,4.

As alterações orgânicas decorrentes do envelhecimento, somadas aos abusos, ao desuso e às condições de vida, impõem comprometimentos típicos desta fase da vida 5 . Sob o aspecto da saúde, essas condições são geralmente marcadas por curso crônico, incapacitante, dependente de cuidados especializados e alto custo, caracterizando o processo de transição epidemiológica que ocorre em conjunto com as transformações demográficas, sociais, econômicas, psicológicas e culturais 6,7

A Organização Mundial da Saúde (OMS) em 2005 indicou os três pilares da estrutura política para o envelhecimento ativo: participação, saúde e segurança, que devem ser observados para que os idosos participem da sociedade de acordo com 
as suas necessidades, desejos e capacidades 8 . No Brasil, em 2003, o Estatuto do Idoso no parágrafo IV indica a necessidade de viabilização de formas alternativas de participação, ocupação e convívio do idoso com as demais gerações ${ }^{9}$. Nesse contexto, os grupos de convivência de idosos vão de encontro à promoção do envelhecimento ativo, com o objetivo de preservação das capacidades e do potencial de desenvolvimento do indivíduo idoso ${ }^{8}$.

O histórico dos grupos de convivência de idosos brasileiros remonta à década de 70, quando o Serviço Social do Comércio (SESC) de São Paulo iniciou um programa para a terceira idade. Desde então, os grupos de convivência de idosos vêm proliferando em clubes, paróquias, associações comunitárias, centros de saúde e instituições de ensino superior 1,10,11,12. Esses grupos realizam atividades variadas, de cunho recreativo, cultural, social, educativo e de promoção da saúde 12. Segundo dados do Departamento de Planejamento da Prefeitura Municipal de Belo Horizonte havia, em 2004, 168 grupos de convivência de idosos cadastrados, registrando 14.023 participantes.

Alguns autores têm caracterizado o perfil social, epidemiológico ou demográfico de Universidades Abertas para a Terceira Idade ou de grupos de convivência de idosos isolados, ou estudado características específicas, como capacidade funcional, qualidade de vida e comorbidades de seus participantes 1,5,13,14. Entretanto, não foram encontrados na revisão de literatura realizada trabalhos que caracterizem os grupos de convivência de idosos em relação aos participantes, locais em que se reúnem, coordenadores e atividades oferecidas.

O levantamento do perfil dos idosos que participam de grupos de convivência de idosos poderia favorecer o direcionamento de ações tanto no plano da saúde quanto nos aspectos físicos, sociais, emocionais, culturais e econômicos; além de delinear as necessidades de adequação destes locais e de suas atividades para o público freqüentador ${ }^{6}$. Adicionalmente, o conhecimento das características dos idosos que freqüentam esses locais poderia contribuir para ações visando à participação de mais idosos. Do ponto de vista da saúde pública, investir na qualidade de vida de idosos da comunidade, para mantê-los em uma situação de envelhecimento ativo, deveria ser considerado fundamental para reduzir a necessidade de investimentos em prevenções secundária e terciária, em tratamentos, reabilitação e institucionalização 6,8.

Portanto, o objetivo deste estudo foi traçar o perfil epidemiológico, sócio-familiar, funcional e econômico dos idosos freqüentadores de gru- pos de convivência de idosos na cidade de Belo Horizonte, além de estudar as associações destes dados entre si.

\section{Métodos}

Foi realizado um estudo de corte transversal exploratório, em que participaram 197 idosos de ambos os sexos, freqüentadores de grupos de convivência de idosos na cidade de Belo Horizonte, Minas Gerais, Brasil. Com base em um cadastro fornecido pelo Departamento de Planejamento da Prefeitura Municipal dessa cidade em 2004, com um total de 168 grupos de convivência de idosos, foram selecionados aleatoriamente 18 destes grupos de convivência, sendo dois de cada uma das nove regionais da cidade (Barreiro, Centro-sul, Leste, Nordeste, Noroeste, Norte, Oeste, Pampulha e Venda Nova), e de cada grupo foram selecionados por conveniência 11 idosos para participarem de entrevista.

Os critérios de inclusão foram: idade igual ou superior a 60 anos; freqüentar o grupo de convivência há no mínimo três meses e aceitar responder ao questionário proposto preenchendo o Termo de Consentimento Livre e Esclarecido. Foram excluídos os idosos incapazes de responderem às perguntas, assim como aqueles que obtiveram desempenho inferior ao esperado para a sua escolaridade no Mini-Exame do Estado Mental (MEEM) - versão para uso no Brasil, com pontos de corte de acordo com a escolaridade 15,16.

O questionário aplicado continha 50 perguntas sobre informações gerais, saúde física, utilização de serviços médicos e dentários, atividades da vida diária, recursos sociais, recursos econômicos e saúde mental. A maioria das questões era fechada e algumas delas continham espaços para anotação de respostas abertas e comentários (nome de medicamentos, por exemplo). Esse questionário foi desenvolvido pelos pesquisadores, tendo como referência o Brazilian Old Age Schedule (BOAS) 17, que é um questionário multidimensional para a população idosa brasileira, já sendo utilizado em uma série de outros estudos semelhantes a este 18,19,20,21,22. O BOAS foi desenvolvido por Veras et al. 17 e adaptado para este estudo devido à sua grande extensão, com demanda de tempo inviável, além da necessidade de inclusão de perguntas que possibilitassem mais informações sobre os freqüentadores dos grupos de idosos. Foram acrescentadas perguntas sobre o tempo de participação nos grupos de convivência de idosos e como eles iam até o grupo de convivência (meio de transporte e acompanhantes). As perguntas que se referiam à avaliação do humor foram substituídas pela Escala de Depres- 
são Geriátrica (GDS), instrumento desenvolvido por Yesavage et al. 23 , traduzida e validada para o Brasil por Almeida \& Almeida 24 em 1999, com boas propriedades psicométricas para a versão reduzida de 15 itens. Trata-se de um questionário com respostas dicotômicas, que sugere a presença de sintomas depressivos, tendo como ponto de corte 5 para ausência de sintomas depressivos e 6 para presença destes sintomas. Como os idosos com possível déficit cognitivo foram excluídos com base no resultado do MEEM, as questões do BOAS referentes ao estado cognitivo do idoso foram retiradas.

Os dados foram coletados por meio de entrevista, com preenchimento de questionário semiestruturado. As entrevistas foram realizadas nos próprios locais de reunião dos grupos de convivência de idosos, após agendamento prévio e autorização por escrito do coordenador do grupo. Para favorecer a confiabilidade das respostas, o ambiente foi organizado de forma a permitir a privacidade da entrevista e foi solicitado ao idoso que estivesse só.

As perguntas foram sempre realizadas pelos examinadores, que informavam e sinalizavam as possibilidades de resposta, considerando a chance dos idosos serem analfabetos ou terem dificuldade para a leitura, e visando a reduzir o viés de erro de comparação entre questionários auto-aplicados e aplicados pelo avaliador. O tempo gasto para responder ao questionário foi de aproximadamente 30 minutos e o mesmo foi aplicado integralmente em um único encontro. Os três examinadores foram submetidos a treinamento prévio para aplicarem o MEEM e o questionário de caracterização dos grupos de convivência de idosos.

Foi realizado cálculo de tamanho de amostra, considerando-se um intervalo de 95\% de confiança, um nível de significância de $5 \%$ e uma margem de erro estimado de $7 \%$, tendo como referência o número total de freqüentadores de grupos de convivência de idosos no cadastro fornecido pela Prefeitura Municipal de Belo Horizonte em 2004, que indicou a necessidade de 197 idosos. Para a caracterização dos freqüentadores dos grupos de convivência de idosos, foi realizada análise descritiva, baseada em medidas de tendência central, dispersão e freqüência. Como havia a presença de variáveis categóricas, com mais de três categorias de resposta, as associações entre as variáveis pesquisadas foram analisadas pelo teste não paramétrico de Kruskal Wallis. Para todas as análises realizadas adotouse um nível de significância de 5\%. Os dados foram analisados no programa estatístico Minitab versão 13.0 (Minitab Inc., State College, Estados Unidos).
Esta pesquisa foi aprovada pelo Comitê de Ética em Pesquisa do Centro Universitário de Belo Horizonte (062/04) e a todos os grupos participantes foi oferecida palestra gratuita sobre temas relacionados à saúde do idoso.

\section{Resultados}

As características demográficas dos idosos freqüentadores dos grupos de convivência de idosos estão descritas na Tabela 1, tendo sido observado predomínio de mulheres $(86,29 \%)$, viúvas $(49,75 \%)$, na faixa etária de 65 a 74 anos $(65,49 \%$, média de 71,66 \pm 6.8 anos) e escolaridade primária (45,69\%). No arranjo domiciliar, apenas $1,02 \%$ dos idosos informou residir sozinhos. Em relação às pessoas com as quais os idosos residiam, $34,01 \%$ moravam com as filhas; $31,98 \%$ com filhos; $28,43 \%$ com esposo ou companheiro; $25,89 \%$ com netos; $13,71 \%$ com outros parentes; $7,61 \%$ com irmãos; $2,54 \%$ com os pais e $1,52 \%$ com empregados e amigos.

Sobre o aspecto da saúde, a freqüência de queixas relatadas de acordo com os sistemas corporais está descrita na Figura 1, tendo havido predomínio de sintomas cardíacos $(60,71 \%)$, músculo-esqueléticos $(44,67 \%)$ e distúrbios circulatórios $(40,10 \%)$.

A maioria dos idosos $(85,28 \%)$ referiu pelo menos uma doença. Em relação à autopercepção da saúde, 51,27\% consideraram sua saúde boa, enquanto $7,11 \%$ a classificaram como péssima. Quanto à utilização de medicamentos, 89,85\% dos entrevistados faziam uso de pelo menos uma droga, cujas indicações mais prevalentes foram: hipertensão arterial sistêmica $(89,84 \%)$, diabetes mellitus $(88,5 \%)$, cardiopatias $(53,1 \%)$ e osteoporose $(12,39 \%)$.

Com relação às síndromes geriátricas, 56,85\% relataram perda urinária, com a seguinte freqüência de ocorrência: 1 ou 2 vezes por dia $(10,10 \%)$; mais de 2 por dia (7,5\%); 1 ou 2 vezes por semana (5,48\%); 1 ou 2 vezes por mês (3,75\%); mais de 2 vezes por semana $(1,73 \%)$; mais de 2 vezes por mês $(1,44 \%)$. Dos idosos entrevistados, 35,03\% relataram quedas nos últimos 12 meses, dos quais $16,75 \%$ tiveram como conseqüência lesão física leve. Na GDS, $24,37 \%$ dos idosos foram classificados como apresentando sinais sugestivos de depressão.

Do total de idosos entrevistados, 65,99\% utilizavam a instituição pública de saúde (SUS). Em relação às consultas nos últimos três meses, 82,23\% fizeramconsultasmédicas; $71,57 \%$ fizeram exames clínicos; $14,21 \%$ realizaram tratamento fisioterápico; $12,69 \%$ foram ao dentista e 5,08\% foram hospitalizados. Quando questionados 
Características demográficas dos idosos participantes de grupos de convivência. Belo Horizonte, Minas Gerais, Brasil.

\begin{tabular}{lcc}
\hline Variável & $\mathbf{n}$ & $\%$ \\
\hline Idade (anos) & & \\
$60-64$ & 27 & 13,71 \\
$65-69$ & 55 & 27,92 \\
$70-74$ & 47 & 23,86 \\
$75-79$ & 42 & 21,32 \\
$>80$ & 26 & 13,20 \\
Sexo & & \\
Feminino & 170 & 86,29 \\
Masculino & 27 & 13,71 \\
Estado civil & & \\
Casado/Morando junto & 53 & 26,90 \\
Viúvo & 98 & 49,75 \\
$\quad$ Divorciado/Separado & 20 & 10,15 \\
Nunca casou & 26 & 13,20 \\
Escolaridade & & 36,55 \\
Nenhuma & 72 & 45,69 \\
Primário & 90 & 7,11 \\
Primeiro grau & 14 & 7,61 \\
Segundo grau & 15 & 3,05 \\
Curso superior & 6 & 1,02 \\
Arranjo domiciliar & & 98,98 \\
Mora sozinho & 2 & \\
Mora acompanhado & 195 & \\
\hline
\end{tabular}

Figura 1

Relato de sintomas apresentados pelos idosos freqüentadores de grupos de convivência. Belo Horizonte, Minas Gerais, Brasil.

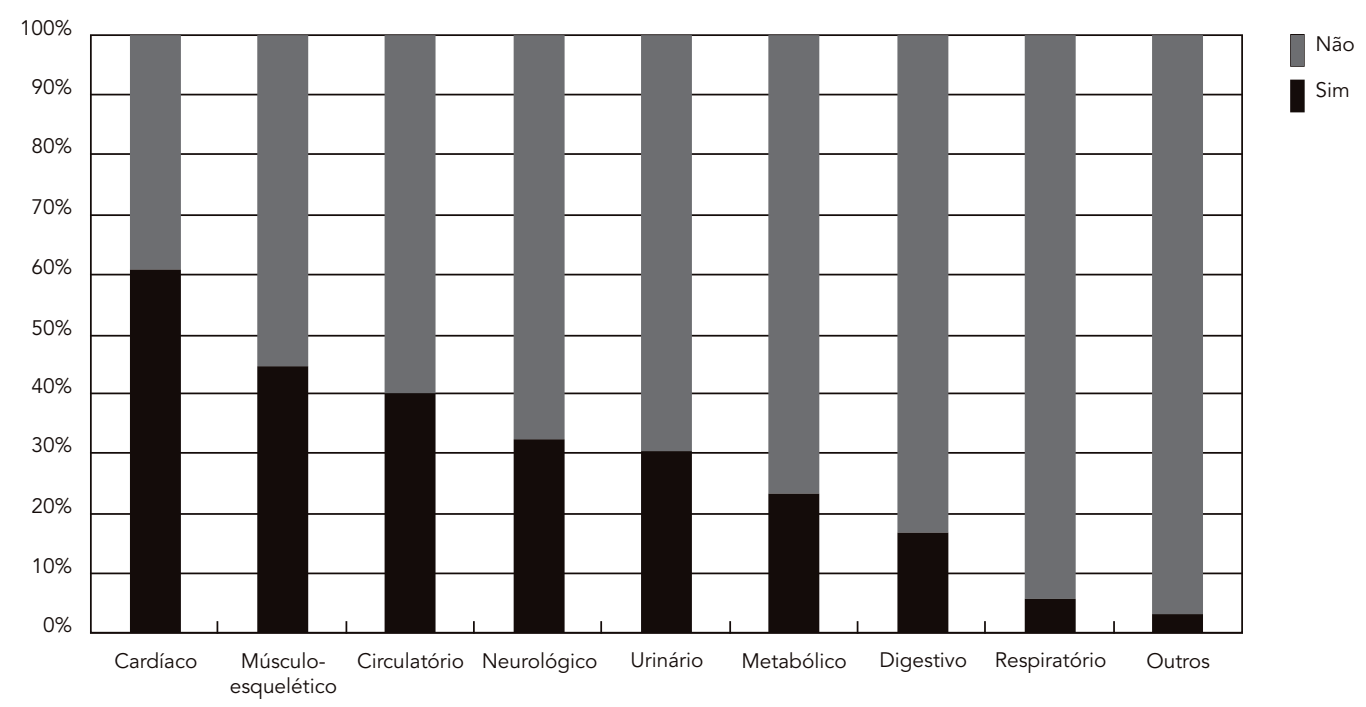


sobre as pessoas que poderiam dispensar-lhes cuidados caso viessem a adoecer ou ficar incapacitados, $46,7 \%$ contariam com as filhas ou filhos e $6,6 \%$ não saberiam com quem contar. Os dados referentes ao perfil epidemiológico dos idosos entrevistados podem ser melhor visualizados na Tabela 2.
Com relação ao perfil funcional dos idosos que participam dos grupos de convivência de idosos, $87,82 \%$ utilizavam prótese dentária e $87,31 \%$ óculos e/ou lentes de contato. Quase a totalidade $(94,41 \%)$ dos idosos entrevistados relatou independência para a deambulação e apenas 2,54\% referiram uso de bengalas. A maioria

Tabela 2

Perfil epidemiológico dos idosos entrevistados nos grupos de convivência. Belo Horizonte, Minas Gerais, Brasil.

\begin{tabular}{|c|c|c|}
\hline Variável & $\mathbf{n}$ & $\%$ \\
\hline \multicolumn{3}{|l|}{ Autopercepção de saúde } \\
\hline Boa & 101 & 51,27 \\
\hline Ruim & 42 & 21,32 \\
\hline Ótima & 39 & 19,80 \\
\hline Péssima & 14 & 7,11 \\
\hline Não sabe/Não respondeu & 1 & 0,51 \\
\hline \multicolumn{3}{|l|}{ Uso de medicamentos } \\
\hline $\operatorname{Sim}$ & 177 & 89,85 \\
\hline Não & 20 & 10,15 \\
\hline \multicolumn{3}{|l|}{ Auto-relato de perda de urina } \\
\hline Sim & 112 & 56,85 \\
\hline Não & 84 & 42,40 \\
\hline Não sabe/Não respondeu & 1 & 0,51 \\
\hline \multicolumn{3}{|l|}{ Freqüência da perda urinária } \\
\hline Não se aplica & 53 & 26,83 \\
\hline 1 ou 2 por dia & 20 & 10,10 \\
\hline Mais de 2 por dia & 15 & 7,50 \\
\hline 1 ou 2 por semana & 11 & 5,48 \\
\hline 1 ou 2 por mês & 7 & 3,75 \\
\hline Mais de 2 por semana & 3 & 1,73 \\
\hline Mais de 2 por mês & 3 & 1,44 \\
\hline \multicolumn{3}{|l|}{ Local de atendimento de saúde } \\
\hline Instituição pública & 130 & 65,99 \\
\hline Plano de saúde & 61 & 30,96 \\
\hline Clinica particular & 4 & 2,03 \\
\hline Ninguém/Muito tempo & 1 & 0,51 \\
\hline Não sabe/Não respondeu & 1 & 0,51 \\
\hline \multicolumn{3}{|c|}{ Tratamento de saúde nos últimos 3 meses } \\
\hline Consultório médico & 162 & 82,23 \\
\hline Exames clínicos & 141 & 71,57 \\
\hline Tratamento fisioterápico & 28 & 14,21 \\
\hline Dentista & 25 & 12,69 \\
\hline Emergência & 14 & 7,11 \\
\hline Hospital/Clínicas para medicação & 12 & 6,09 \\
\hline Hospital/Clínicas para internação & 10 & 5,08 \\
\hline \multicolumn{3}{|c|}{ Pessoa indicada para prestar cuidados de saúde } \\
\hline Filha/Filho & 92 & 46,70 \\
\hline Esposo/Companheiro & 51 & 25,89 \\
\hline Outro da família & 39 & 19,80 \\
\hline Outro não da família & 38 & 19,29 \\
\hline Nenhum & 20 & 10,15 \\
\hline Não sabe/Não respondeu & 13 & 6,60 \\
\hline
\end{tabular}


da população estudada $(85,34 \%)$ relatou independência para as atividades básicas da vida diária e para as atividades instrumentais do dia-adia. Entre os idosos que se queixaram de algum tipo de dependência para essas atividades, as tarefas com maior relato de limitação para sua execução foram: sair dirigindo o próprio carro $(85,28 \%)$; cortar as unhas dos pés $(26,4 \%)$; ir ao banheiro em tempo (15,74\%); e sair utilizando transporte $(15,23 \%)$. Dentre os entrevistados apenas $47,7 \%$ não realizavam uma tarefa. O perfil funcional dos idosos entrevistados está ilustrado na Tabela 3.

Quanto ao aspecto social, a maioria (68,53\%) informou satisfação com o relacionamento mantido com as pessoas com quem morava e $94,42 \%$ encontravam-se satisfeitos com o relacionamento entre amigos.

O perfil econômico dos idosos entrevistados mostrou que 90,36\% não apresentavam atividade produtiva remunerada. Quanto à renda mensal, 65,99\% dos participantes relataram renda familiar de meio a um salário mínimo. Para as necessidades básicas, 40,1\% dos idosos referiram que o que ganhavam dava a conta certa e 13,2\% avaliaram que dava e sobrava. Apesar disso, 40\% informaram fornecer ajuda financeira e moradia para a família.

Em relação à participação dos idosos no grupo de convivência, a maioria $(41,62 \%)$ freqüentava os grupos há mais de 5 anos e apenas 4,21\% há menos de 1 ano; 64,97\% dos idosos informaram ir andando até os grupos de convivência e 73,6\% vão sozinhos até os locais de reunião.

Quanto às associações entre as variáveis estudadas, observou-se associação significativa entre autopercepção de saúde com quedas $(p=0,002)$ e incontinência urinaria $(p=0,010)$; quedas com problemas neurológicos $(\mathrm{p}=0,035)$, GDS ( $\mathrm{p}=<0,05)$, sintomas músculo-esqueléticos ( $p=0,048)$ e digestivos ( $p=0,010)$; incontinência urinária com GDS ( $\mathrm{p}=0,009)$, atividades da vida diária $(\mathrm{p}<0,05)$ e quedas $(\mathrm{p}=0,021)$; de satisfação com a família e estado civil $(p=0,003)$. As associações significativas estão ilustradas na Tabela 4.

\section{Discussão}

Com relação às características demográficas encontradas, este estudo verificou que a maioria dos idosos participantes dos grupos de convivência de idosos era do sexo feminino, viúvas, com idades entre 65-74 anos, renda mensal de meio a um salário mínimo, escolaridade primária e moravam acompanhados, dados que estão de acordo com uma série de estudos tanto de base populacional quanto com populações específicas de idosos 1,5,6,25,26, assim como com os dados dos idosos brasileiros levantados pelo IBGE 2 .

Apesar da feminilização do envelhecimento, a proporção entre mulheres e homens participando dos grupos de convivência de idosos foi maior do que esta relação para a população idosa brasileira 1. Embora o motivo para essa diferença não tenha sido pesquisado, possíveis justificativas podem se relacionar à persistência da viuvez entre as mulheres da terceira idade enquanto os homens tendem a assumir novos casamentos, e à resistência masculina em encontrar novas atividades após a aposentadoria e em engajar-se em atividades de cunho cultural, educacional e lúdico 1,4,5,6. Portanto, ações destinadas aos grupos de convivência de idosos devem considerar o universo feminino na terceira idade e suas peculiaridades, ao mesmo tempo em que considerem alternativas que atraiam os homens, favorecendo sua integração social, informação, lazer e qualidade de vida.

A pequena presença de idosos entre 60 e 69 anos pode se associar à sua participação no mercado de trabalho, com menor tempo livre para atividades lúdicas, ou a uma fuga do estereótipo de idoso. Por outro lado, a baixa participação de idosos com mais de 80 anos pode decorrer de maiores graus de dependência e comorbidades com o aumento da idade, limitando o acesso e a participação nos grupos de convivência de idosos 1,28. Entretanto, essas possíveis justificativas não foram objetos desta pesquisa.

A baixa escolaridade encontrada está de acordo com uma série de estudos de base populacional 1,2,5,6,25,26, mas pode se associar também ao tipo de atividade oferecida nos grupos de convivência de idosos, que não requer escolaridade mínima para participação e entendimento. Assim, no desenvolvimento de atividades para os grupos de convivência de idosos, não só devese considerar sua adequação para a baixa escolaridade dos idosos, mas pensar na proposição de programas que possibilitem novas formas de acesso à educação formal e informal, com metodologia adequada às necessidades deste público.

No arranjo domiciliar, quase a totalidade (98,98\%) dos idosos entrevistados residia acompanhado e destes, $65,99 \%$ residiam com os filhos, dado confirmado pelo estudo de Pereira et al. 4 , em que a maioria dos idosos nos países em desenvolvimento vivia com seus filhos. Essa informação indica que os grupos de convivência de idosos poderiam desenvolver atividades de caráter intergeracional e envolver as famílias nos programas, buscando melhor adaptação do idoso à família e mais informações gerontológicas. 
Perfil funcional dos idosos participantes de grupos de convivência. Belo Horizonte, Minas Gerais, Brasil.

\begin{tabular}{|c|c|c|c|c|}
\hline \multirow[t]{2}{*}{ Variável } & \multicolumn{2}{|c|}{ Sim } & \multicolumn{2}{|c|}{ Não } \\
\hline & $\mathbf{n}$ & $\%$ & $\mathbf{n}$ & $\%$ \\
\hline Sair dirigindo & 29 & 14,72 & 168 & 85,28 \\
\hline Cortar unhas dos pés & 145 & 73,60 & 52 & 26,40 \\
\hline Ir ao banheiro em tempo & 166 & 84,26 & 31 & 15,74 \\
\hline Sair utilizando transporte & 167 & 84,77 & 30 & 15,23 \\
\hline Arrumar casa/cama & 168 & 85,28 & 29 & 14,72 \\
\hline Preparar refeição & 177 & 89,85 & 20 & 10,15 \\
\hline Sair de casa (perto) & 180 & 91,37 & 17 & 8,63 \\
\hline Subir/Descer escadas & 182 & 92,39 & 15 & 7,61 \\
\hline Comer refeição & 187 & 94,42 & 11 & 5,58 \\
\hline Tomar remédios & 187 & 94,42 & 11 & 5,58 \\
\hline Vestir-se/Despir-se & 187 & 94,42 & 11 & 5,58 \\
\hline Caminhar no plano & 187 & 94,42 & 11 & 5,58 \\
\hline Deitar/Levantar da cama & 187 & 94,42 & 11 & 5,58 \\
\hline Pentear cabelos & 188 & 95,43 & 9 & 4,57 \\
\hline Tomar banho & 189 & 95,94 & 8 & 4,06 \\
\hline
\end{tabular}

Tabela 4

Associações estatisticamente significativas $(p \leq 0,05)$ entre as variáveis pesquisadas. Belo Horizonte, Minas Gerais, Brasil.

\begin{tabular}{llc}
\hline Variável A & Variável B (categoria predominante) & Valor de p \\
\hline \multirow{2}{*}{ Autopercepção de saúde } & Quedas & 0,002 \\
Ausência de quedas & Incontinência urinaria & 0,010 \\
& Problemas neurológicos & 0,035 \\
Quedas & GDS & 0,000 \\
& Problemas músculo-esqueléticos & 0,048 \\
Incontinência urinária & Problemas digestivos & 0,010 \\
& GDS & 0,009 \\
Satisfação com a família & Atividades da vida diária & 0,050 \\
& Quedas & 0,021 \\
\hline
\end{tabular}

GDS: Escala de Depressão Geriátrica.

Com relação ao perfil epidemiológico, a maioria dos entrevistados considerou sua saúde boa e ótima, apesar da grande maioria também referir pelo menos uma doença. Pode-se inferir que o uso de serviços de saúde e de medicamentos pela maior parte dos idosos entrevistados indique provável controle e tratamento das doenças associadas com menor efeito das comorbidades. O bom nível de independência para atividades básicas da vida diária e atividades instrumentais do dia-a-dia confirma essa possi- bilidade. Estudos de base populacional realizados em 2003, encontraram resultados conflitantes quanto à autopercepção de saúde de idosos comunitários: Araújo et al. 12 encontraram boa percepção de saúde, enquanto Lima-Costa et al. 26 verificaram um nível de percepção de saúde regular. Esses resultados sugerem que os grupos de convivência de idosos podem ser considerados importantes veículos para informações nos três níveis de saúde do idoso, visando a informar sobre a prevenção e controle dos principais pro- 
blemas de saúde do envelhecimento. A inclusão dos grupos de convivência nas campanhas e medidas de saúde pública voltadas para o idoso, também não deve ser desconsiderada.

O uso de medicamentos relatado pelos participantes foi semelhante ao encontrado por Loyola et al. 28 , que investigou o consumo de medicamentos e fatores associados em 1.598 idosos de Belo Horizonte, encontrando freqüência de uso de medicamentos de $72,1 \%$, predominando as drogas com ação sobre o sistema cardiovascular. Assim, o uso de medicamentos não parece ser um fator limitante da participação em grupos de convivência de idosos.

Quanto às síndromes geriátricas, o índice de incontinência urinária encontrado neste estudo pode ser considerado alto (56,85\%) em comparação aos valores verificados em pesquisas com populações diferentes ${ }^{30}$. Esse achado pode sugerir que os idosos se sentiram à vontade para falar da incontinência urinária ou pode se associar à forma de perguntar, que considerou qualquer perda urinária. De qualquer maneira, parece que a presença da incontinência urinária não limita a participação do idoso nos grupos de convivência. Esse pode ser um dos temas de saúde relevantes para abordagem e discussão com os idosos nos grupos de convivência de idosos. Neste estudo, $35,03 \%$ dos entrevistados sofreram quedas nos últimos 12 meses, e destes, 16,75\% sofreram lesão física leve em decorrência da mesma. De acordo com Fabrício et al. 31, 30\% das pessoas com 65 anos ou mais cai a cada ano. Para Perracini et al. ${ }^{32}$, as chances de quedas recorrentes aumentam entre mulheres, viúvos, solteiros ou desquitados, historia prévia de fraturas, grau de comprometimento nas atividades da vida diária e entre os que não referem leitura como atividade de lazer. A freqüência de quedas neste estudo não deve ser negligenciada e pode indicar a necessidade de adequação dos fatores ambientais de risco para quedas nos grupos de convivência de idosos, e a abordagem dos fatores intrínsecos de risco de quedas entre os idosos nos grupos de convivência.

Em relação à utilização dos serviços de saúde, a maioria $(65,99 \%)$ dos idosos entrevistados estava satisfeita com o SUS, embora não recebam atendimento especializado em geriatria e gerontologia. Segundo Romero et al. 33 , fatores como classe social, escolaridade, etnia ou raça e renda afetam a utilização dos serviços de saúde.

A grande utilização de serviços médicos e exames clínicos e a pequena realização de serviços complementares de reabilitação, pode estar associada à indisponibilidade deste tipo de serviço no SUS, à baixa indicação dos mesmos pelos médicos das unidades básicas ou ao nível funcio- nal dos entrevistados, que ainda não necessitam da reabilitação mas poderiam se beneficiar da prevenção nestas áreas.

O baixo uso de dispositivos de auxílio para a marcha confirma a independência funcional dos idosos participantes de grupos de convivência de idosos, e sugere que a acessibilidade aos grupos de convivência pode ser um fator limitante à participação de idosos mais dependentes nos grupos de convivência de idosos, questionamento que não foi incluído nesta pesquisa. Entre as atividades básicas da vida diária e atividades instrumentais do dia-a-dia mais limitadas estavam dirigir o próprio carro, que pode decorrer da ausência de posse de veículo ou de habilitação para dirigir; e cortar as unhas dos pés, que pode ser uma tarefa difícil por limitações músculo-esqueléticas ou desnecessária pelo uso de serviços de pedicures. O bom nível funcional entre os idosos nos grupos de convivência de idosos deve levar à reflexão sobre a inclusão de idosos mais dependentes, garantindo o acesso dos mesmos a estes locais, bem como atividades que possibilitem sua participação.

As filhas foram as pessoas mais indicadas para prestar cuidados diante de uma possível doença ou incapacidade, fato que está de acordo com os estudos de Barreto et al. ${ }^{1}$ e Silveira et al. 34 . Para Giacomin et al. 35 , o cuidado do idoso dependente sempre coube à família brasileira, porém, a ausência de especialização, a redução do tamanho das famílias e a participação das mulheres no mercado de trabalho podem gerar uma maior sobrecarga às filhas. Esses resultados confirmam a necessidade de inclusão das famílias no universo dos grupos de convivência de idosos.

A maioria dos participantes relatou satisfação com a interação social com a família, amigos e vizinhos. Resultado semelhante foi encontrado por Barreto et al. 1, que afirmam que as relações entre os participantes dos grupos de convivência de idosos fortalecem a experiência de grupo e favorecem a ampliação das relações interpessoais, além de serem tão importantes quanto o apoio familiar. Não foi objetivo desta pesquisa avaliar se a participação no grupos de convivência de idosos favorece uma melhor interação social.

A maioria dos idosos entrevistados referiu renda de meio a um salário mínimo, conforme também foi relatado por Camargos et al. 36 e Azevedo et al. 27. Ainda assim, os idosos informaram oferecer mais assistência financeira às famílias do que a família para os mesmos. Vários idosos referiram contribuir com as famílias cuidando das crianças, o que pode ser explicado pela disponibilidade de tempo que estes idosos apresentam e favorecer maior contato intergeracional. 
$\mathrm{O}$ fato de que a grande maioria dos idosos freqüentava os grupos de convivência de idosos há mais de cinco anos, sugere que os mesmos estão encontrando o que buscam nestes locais e confirmam a possibilidade de utilização dos grupos de convivência como veículos para aumentar a qualidade de vida e a oferta de serviços aos idosos.

O índice de idosos deprimidos (24,3\%) foi semelhante ao encontrado por Lebrão et al. 37 em um estudo de base populacional, e ao de Leite et al. ${ }^{38}$ que teve como objetivo identificar a presença de depressão em idosos que freqüentavam o programa Universidade Aberta à Terceira Idade da Universidade Federal de Pernambuco. Com base nesses dados pode-se questionar se a participação nos grupos de convivência de idosos reduz a depressão ou se idosos deprimidos não freqüentam grupos de convivência, o que pode ser pesquisado em novos estudos.

As limitações da pesquisa incluem a ausência de consideração da densidade de grupos por regional da cidade no cálculo amostral, a escolha dos idosos participantes por conveniência e não ter considerado a assiduidade dos idosos entrevistados. Embora tenha sido realizada seleção aleatória dos grupos de convivência de idosos e recrutamento dos mesmos em todas as regionais de Belo Horizonte, a generalização dos resultados para outros municípios e realidades sociais, econômicas e culturais deve ser cautelosa.

\section{Conclusão}

Os resultados indicam que os grupos de convivência de idosos na cidade de Belo Horizonte são freqüentados principalmente por mulheres, viúvas e de baixa escolaridade. Embora a maioria dos idosos entrevistados nesses locais tenha relatado pelo menos uma co-morbidade, uso de drogas e consultas médicas freqüentes, os mesmos consideraram sua saúde boa. A incontinência urinária foi a síndrome geriátrica mais freqüente, a ocorrência de quedas só foi citada por cerca de um terço dos participantes, sendo que a maioria deles não apresentou sinais sugestivos de depressão. Os idosos que participam de grupos de convivência de idosos são de baixa renda, independentes para deambulação e para as atividades da vida diária e atividades instrumentais do dia-a-dia, e relatam satisfação com seus relacionamentos sociais. As alterações de saúde encontradas reforçam a incidência das síndromes geriátricas e a necessidade de atenções primária, secundária e terciária específicas para os idosos. Esse perfil social e epidemiológico indica que os grupos de convivência de idosos podem ser importantes veículos para que as ações de saúde atinjam um número significativo de idosos. Essa caracterização deve ser considerada na programação e implementação das medidas de saúde e geração de renda para idosos da comunidade, e para a adequação desses locais para que possam não só atender aos participantes, mas também a outros idosos que poderão se beneficiar de suas atividades.

\section{Resumo}

Este trabalho caracterizou os idosos freqüentadores de grupos de convivência sob os aspectos físico, social, psicológico e econômico. Realizou-se estudo descritivo transversal com 197 idosos, utilizando o Brazilian Old Age Schedule modificado, com perguntas sobre saúde física, serviços médicos, atividades da vida diária, recursos sociais, econômicos e saúde mental. A maioria dos participantes era mulher, viúva, com escolaridade primária, média de idade de 71,66 $\pm 6,8$ anos; $51,27 \%$ consideraram sua saúde boa, 85,28\% referiram pelo menos uma doença; $63,96 \%$ não relataram quedas no último ano e 56,85\% relataram incontinência urinária. A maioria freqüentava os grupos de convivência de idosos há mais de cinco anos; 65,99\% apresentaram renda de até um salário mínimo e 24,37\% apresentaram sintomas depressivos. Houve associação da autopercepção de saúde com quedas e incontinência urinária; de quedas com problemas neurológicos, músculo-esqueléticos, digestivos e sintomas depressivos, de incontinência urinária com sintomas depressivos e atividades da vida diária e de satisfação com a família e estado civil. As alterações de saúde encontradas reforçam o perfil epidemiológico dos idosos e podem contribuir para o direcionamento de ações para a saúde coletiva dos mesmos.

Envelhecimento; Centros de Convivência e Lazer; Saúde do Idoso 


\section{Colaboradores}

P. L. C. Borges colaborou na elaboração do projeto de pesquisa, coleta e tabulação de dados, revisão da literatura e preparação do manuscrito para publicação. R. P. Bretas e S. F. Azevedo participaram ativamente de todas as etapas da pesquisa, à exceção da revisão final do artigo para publicação. J. M. M. Barbosa contribuiu em todas as etapas da pesquisa e participou na revisão final do manuscrito.

\section{Referências}

1. Barreto KML, Carvalho EMF, Falcão IV, Lessa FJD, Leite VMM. Perfil sócio epidemiológico demográfico das mulheres idosas da Universidade Aberta à Terceira Idade no Estado de Pernambuco. Rev Bras Saúde Matern Infant 2003; 3:339-54.

2. Instituto Brasileiro de Geografia e Estatística. Perfil dos idosos responsáveis pelos domicílios no Brasil 2000. http://www.ibge.gov.br (acessado em 10/ Out/2004).

3. Zahreddine D, Rigotti JIR. O processo de envelhecimento populacional em Belo Horizonte: análise e mapeamento dos anos 1991 e 2000. In: Anais do XV Encontro de Estudos Populacionais. Caxambu: Associação Brasileira de Estudos Populacionais; 2006. p. 18-22.

4. Pereira RS, Curioni CC, Veras R. Perfil demográfico da população idosa no Brasil e no Rio de Janeiro em 2002. Texto Envelhecimento 2003; 6:43-59.

5. Ramos LR. Fatores determinantes do envelhecimento saudável em idosos residentes em centro urbano: Projeto Epidoso, São Paulo. Cad Saúde Pública 2003; 19:793-7.

6. Chaimowicz F. A saúde dos idosos brasileiros às vésperas do século XXI: problemas, projeções e alternativas. Rev Saúde Pública 1997; 31:184-200.

7. Carvalho JAM, Garcia RA. O envelhecimento da população brasileira: um enfoque demográfico. Cad Saúde Pública 2003; 19:725-33.
8. Organização Pan-Americana da Saúde. Envelhecimento ativo: uma política de saúde. Brasília: Organização Pan-Americana da Saúde OPAS; 2005.

9. Parecer no. 1301. Redação final do Projeto de Lei da Câmara no. 57. Dispõe sobre o Estatuto do Idoso e outras providências. Diário Oficial da União 2003; 13 set.

10. Brito FC, Ramos LR. Gerontologia: a velhice e o envelhecimento em visão globalizada. In: Papaleo Netto M, organizador. Serviços de atenção à saúde do idoso. São Paulo: Editora Atheneu; 2000. p. 394-403.

11. Garrido R, Menezes PR. O Brasil está envelhecendo: boas e más notícias por uma perspectiva epidemiológica. Rev Bras Psiquiatr 2002; 24 Suppl 1:3-6.

12. Araújo LF, Carvalho VML. Velhices: estudo comparativo das representações sociais entre idosos de grupo de convivência. Textos Envelhecimento 2004; 6:10-22.

13. Veras RP, Caldas CP. Promovendo a saúde e a cidadania do idoso: o movimento das universidades da terceira idade. Ciênc Saúde Coletiva 2004; 9 : 423-32.

14. Leite VMM, Carvalho EMF, Barreto KML, Falcão IV. Depressão e envelhecimento: estudos nos participantes do programa Universidade aberta a terceira idade. Rev Bras Saúde Matern Infant 2006; 6:31-8. 
15. Bruck SMD, Nitrini R, Caramelli P, Bertolucci PHF, Okamoto IH. Sugestões para uso de mini exame do estado mental no Brasil. Arq Neuropsiquiatr 2003; 61:777-81.

16. Bertolucci PH, Brucki SM, Campacci SR, Juliano Y. The Mini-Mental State Examination in a general population: impact of educational status. Arq Neuropsiquiatr 1994; 52:1-7.

17. Veras RP, Souza CAM, Cardoso RF, Milioli R, Silva SD. Pesquisando populações idosas - a importância do instrumento e o treinamento de equipe: uma contribuição metodológica. Rev Saúde Pública 1988; 22:513-8.

18. Pinheiro RS, Travassos C. Estudo da desigualdade na utilização de serviços de saúde por idosos em três regiões da cidade do Rio de Janeiro. Cad Saúde Pública 1999; 15:487-96.

19. Lovisi GM. Avaliação de distúrbios mentais em moradores de albergues públicos das cidades do Rio de Janeiro e de Niterói [Tese de Doutorado]. Rio de Janeiro: Escola Nacional de Saúde Pública, Fundação Oswaldo Cruz; 2000.

20. Scazufca M, Cerqueira ATAR, Menezes PR, Prince M, Vallada HP, Miyazaki MCOS, et al. Investigações epidemiológicas sobre demência nos países em desenvolvimento. Rev Saúde Pública 2002; 36:773-8.

21. Anderson MIP, Assis M, Pacheco LC, Silva EA, Menezes IS, Duarte T, et al. Saúde e qualidade de vida na terceira idade. Textos Envelhecimento 1998; $1: 1-44$.

22. Paiva SOC. Perfil socioeconômico e epidemiológico da população idosa do Distrito Estadual de Fernando de Noronha - PE [Dissertação de Mestrado]. Recife: Centro de Pesquisas Aggeu Magalhães, Fundação Oswaldo Cruz; 2004.

23. Yesavage JA, Brink TL, Rose TL, Lum O, Huang V, Adey MB, et al. Development and validation of a geriatric depression screening scale: a preliminary report. J Psychiatr Res 1983; 17:37-49.

24. Almeida OP, Almeida SA. Confiabilidade da versão brasileira da escala de depressão em geriatria (GDS) versão reduzida. Arq Neuropsiquiatr 1999; 57:421-6.

25. Berquó E. Algumas considerações demográficas sobre o envelhecimento da população no Brasil. In: Anais do I Seminário Internacional "Envelhecimento Populacional: Uma Agenda para o Final do Século". Brasília: Ministério da Previdência e Assistência Social; 1996; p. 13-5.
26. Lima-Costa MF, Barreto S, Giatti L. A situação socioeconômica afeta igualmente a saúde de idosos e adultos mais jovens no Brasil? Um estudo utilizando dados da Pesquisa Nacional por Amostra de Domicílios - PNAD/98. Ciênc Saúde Coletiva 2002; 7:813-24.

27. Azevedo MB, Ruiz T, Barros LVAS. Estudo de sobrevivência de uma coorte de pessoas de 60 anos e mais no Município de Botucatu (SP) - Brasil. Rev Bras Epidemiol 2003; 6:227-36.

28. Loyola Filho AI, Uchoa E, Lima-Costa MF. Estudo epidemiológico de base populacional sobre o uso de medicamentos entre idosos na região Metropolitana de Belo Horizonte, Minas Gerais, Brasil. Cad Saúde Publica 2006; 22:2657-67.

29. Rozenfeld S. Prevalência, fatores associados e mau uso de medicamentos entre idosos: uma revisão. Cad Saúde Publica 2003; 19:717-24.

30. Marques LM. Prevalência e fatores associados à incontinência urinária em idosos residentes na comunidade; Ouro Preto [Dissertação de Mestrado]. Belo Horizonte: Universidade Federal de Minas Gerais; 2005.

31. Fabrício SCC, Rodrigues RAP, Costa MLJ. Causas e conseqüências de quedas de idosos atendidos em hospital público. Rev Saúde Pública 2004; 38:93-9.

32. Parracini MR, Ramos LR. Fatores associados a quedas em uma coorte de idosos residentes na comunidade. Rev Saúde Pública 2002; 36:709-16.

33. Romero DE. Diferenciais de gênero no impacto do arranjo familiar no status de saúde dos idosos brasileiros. Ciênc Saúde Coletiva 2002; 7:777-94.

34. Silveira TM, Caldas CP, Carneiro TF. Cuidando de idosos altamente dependentes. Cad Saúde Pública 2006; 22:1629-38.

35. Giacomin KC, Uchoa E, Lima-Costa MFF. Projeto Bambuí: a experiência do cuidado domiciliário por esposas de idosos dependentes. Cad Saúde Pública 2005; 21:1509-18.

36. Camargos MCS, Machado CJ, Rodrigues RN. A relação entre renda e morar sozinho para idosos paulistanos: 2000. Rev Bras Estud Popul 2007; 24:37-51.

37. Lebrão ML, Laurenti R. Saúde, bem-estar e envelhecimento: o estudo SABE no Município de São Paulo. Rev Bras Epidemiol 2005; 8:121-41.

38. Leite VMM, Carvalho EMF, Barreto KML. Depressão e envelhecimento: estudo nos participantes do Programa Universidade Aberta à Terceira Idade. Rev Bras Saúde Matern Infant 2006; 6:31-8.

Recebido em 26/Fev/2008

Versão final reapresentada em 30/Abr/2008 Aprovado em 05/Mai/2008 